\title{
CUANTIFICACIÓN DE CLORPIRIFOS EN MUESTRAS ACUOSAS POR MICROEXTRACCIÓN LÍQUIDO-LÍQUIDO Y CROMATOGRAFÍA DE GASES CON DETECTOR $\mu$-ECD
}

\section{QUANTIFICATION OF CHLORPYRIFOS IN AQUEOUS SAMPLES BY LIQUID-LIQUID MICROEXTRACTION AND GAS CHROMATOGRAPHY WITH $\mu$-ECD DETECTOR}

\section{Tatiana Garrido Reyes ${ }^{1 *}$, Nicol Campos Escobar ${ }^{1}$ \& Jorge Mendoza Crisosto ${ }^{1}$}

Recibido: 25 de octubre 2021 / Aceptado: 13 de diciembre 2021 DOI 10.26807/ia.v10i1.224

Palabras clave: clorpirifos, cromatografía de gases, microextracción líquido-líquido, química analítica verde.

Keywords: chlorpyrifos, gas chromatography, green analytical chemistry, liquid-liquid microextraction.

1 Universidad de Chile, Facultad de Ciencias Químicas y Farmacéuticas, Departamento de Química Inorgánica y Analítica, Santiago, Chile. (*correspondencia: tgarrido@ciq.uchile.cl, nnicolecampos@gmail.com, jmendoza@ciq.uchile.cl) 


\section{RESUMEN}

La evaluación de contaminantes presentes en el medioambiente es un tema reiterativo a nivel internacional, por ello resulta imperante el desarrollo de nuevas metodologías de extracción de analitos desde matrices como aguas superficiales, con un bajo impacto sobre el medio ambiente. El clorpirifos (CP) es un compuesto organofosforado ampliamente usado para el control de plagas, tóxico para los seres vivos, el cual se puede movilizar desde los suelos a las distintas fuentes de agua. La microextracción líquido-líquido dispersiva (DLLME, por sus siglas en inglés) puede ser considerada como una metodología con características de química analítica verde, por el bajo consumo de solvente y tiempo. El objetivo de este trabajo fue optimizar la DLLME para el análisis de CP en muestras de agua. Para la extracción se usó metanol como agente dispersante y $\mathrm{CS}_{2}$ como agente extractante, evaluándose cinco tiempos de extracción: 5, 10, 15, 20 y 25 minutos. Los extractos fueron analizados por cromatografía de gases con detector de microcaptura electrónica (GC$\mu \mathrm{ECD})$. Los resultados de la evaluación del tiempo de agitación dieron cuenta que, para los tiempos de agitación de 10 y 15 minutos se obtuvo un porcentaje de recuperación de CP de 108 y 88 \%, respectivamente, con un límite de detección y de cuantificación de 18,3 y 22,4 $\mu \mathrm{g} / \mathrm{L}$, respectivamente, y una precisión expresada como desviación estándar relativa menor al 14,2 \%, aceptable para este tipo de extracción. Se puede concluir que la metodología DLLME asociada a GC- $\mu$ ECD es una alternativa adecuada para la cuantificación de CP en matrices acuosas con un bajo impacto medioambiental.

\section{ABSTRACT}

The evaluation of pollutants present in the environment is a reiterative issue at the international level, for this reason the development of new methodologies for the extraction of analytes from matrices such as surface waters, with a low impact on the environment, is imperative. Chlorpyrifos (CP) is an organophosphate compound widely used for pest control, toxic to living beings, which can be mobilized from soils to different water sources. Dispersive li- 
quid-liquid microextraction (DLLME) can be considered as a methodology with characteristics of green analytical chemistry, due to the low consumption of solvent and time. The objective of this work was to optimize the DLLME for the analysis of $\mathrm{CP}$ in water samples. For the extraction, methanol was used as a dispersing agent and $\mathrm{CS}_{2}$ as an extracting agent, evaluating five extraction times: 5, 10, 15, 20 and 25 minutes. The extracts were analyzed by gas chromatography with an electron microcapture detector (GC- $\mu \mathrm{ECD})$. The results of the evaluation of the stirring time showed that, for the stirring times of 10 and 15 minutes, a recovery percentage of $\mathrm{CP}$ of 108 and $88 \%$ was obtained, respectively, with a detection and quantification limit of 18, 3 and $22.4 \mu \mathrm{g} /$ $\mathrm{L}$, respectively, and a precision expressed as a relative standard deviation of less than $14.2 \%$, acceptable for this type of extraction. It can be concluded that the DLLME methodology associated with GC- $\mu$ ECD is a suitable alternative for the quantification of $\mathrm{CP}$ in aqueous matrices with a low environmental impact.

\section{INTRODUCCIÓN}

Los compuestos organofosforados forman parte de los plaguicidas de mayor uso a nivel mundial, dado su bajo costo y gran efectividad, siendo detectados en muestras de aguas subterráneas, aguas superficiales, agua potable, suelos y vegetales (Martínez et al., 2000; Lambropoulou et al., 2002). Entre ellos destaca el clorpirifos [O,O-dietil O-(3,5,6-tricloropiridin-2-il) fosforotioato] (CP), cuya toxicidad puede incrementar en presencia de sus metabolitos, tales como el clorpirifos oxon (CPO) (Figura 1), uno de sus metabolitos producto de su oxidación.
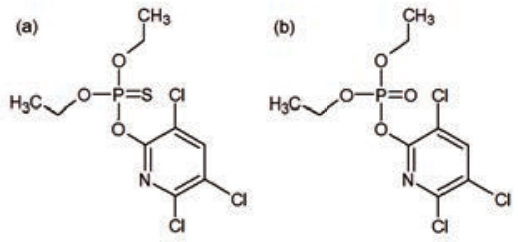

Figura 1. (a) Clorpirifos.

(b) Clorpirifos Oxon 
El CP es un insecticida de amplio uso en el hogar y en agricultura donde, se aplica de forma directa a cosechas. Este compuesto se degrada por acción de la luz solar, bacterias $\mathrm{u}$ otros procesos químicos (ATSDR, 1997). Se ha reportado que su persistencia en el suelo va de unos pocos días hasta 4 años y en vegetales entre 3 a 60 días, aproximadamente, dependiendo de las características fisicoquímicas del suelo; siendo relevante el contenido de materia orgánica presente (Márquez et al., 2010).

Se ha descrito que CP se distribuye un $49,6 \%$ en el suelo, $46,3 \%$ en los sedimentos y $2,3 \%$ en agua, sin embargo, estos rangos varían de acuerdo con la tasa de aplicación, el tipo de ecosistema y factores medioambientales (Mackay, 2001). El CP como contaminante es capaz de movilizarse a través del perfil de suelo y llegar a aguas subterráneas, o bien ser transportado a aguas superficiales. A nivel de toxicidad, el CP es altamente tóxico tanto para peces como para organismos invertebrados (Giesy et al., 1999) y para la vida humana clasifica como un plaguicida de clase II (EPA, 2016; Lazi $\square$ et al., 2012).
Para el análisis de contaminantes en matrices medioambientales, la extracción del analito de interés desde la matriz constituye uno de los pasos críticos en el proceso. En este sentido, se hace necesario evaluar nuevas metodologías de extracción bajo el concepto de la química analítica verde, el cual involucra el desarrollo de metodologías analíticas con una preocupación medioambiental, eliminando o disminuyendo las fuentes de contaminantes con la intención de que los productos y procesos no pongan en peligro cualquier forma de vida. La química analítica verde fomenta el uso de análisis directo, así como la miniaturización, automatización y ahorro tanto de energía como de insumos, reduciendo así los desechos generados y garantizando la seguridad del operador (Armenta et. al., 2019).

La clásica extracción líquido-líquido requiere de prolongados periodos de tiempo, grandes volúmenes de disolventes y múltiples etapas, donde problemas como la emulsión de la muestra dificultan la extracción del analito desde la matriz (Marsin et al., 2011); para mejorar este proceso y disminuir la cantidad de solventes 
empleados la micro-extracción líquido-líquido dispersiva (DLLME, por sus siglas en inglés) puede ser una excelente alternativa. Esta metodología fue desarrollada para pre-concentrar analitos orgánicos desde matrices acuosas y ha sido aplicada a matrices alimenticias, fluidos biológicos y muestras sólidas (Rezaee et al., 2006 and 2010). La DLLME integra en una etapa la extracción y concentración de los analitos, empleando dos fases líquidas inmiscibles: una acuosa (donde se encuentra la muestra) y otra orgánica, (disolvente orgánico) (Martins et al., 2012), a la cual se transfiere el analito de interés para su posterior cuantificación. Dentro de las técnicas más utilizadas para la cuantificación de CP está la cromatografía de gases con detector de microcaptura electrónica (GC- $\mu \mathrm{ECD}$, por sus siglas en inglés).

Según lo descrito resulta de gran importancia contar con metodologías de extracción amigables con el medio ambiente y con adecuadas cifras de mérito que permitan realizar la determinación de contaminantes como CP en muestras de aguas y poder evaluar su movilidad en el medioambiente, siendo este el objetivo perseguido en esta investigación.

\section{MATERIALES Y MÉTODOS}

Se preparó una curva de calibración entre 25 y $400 \mu \mathrm{g} / \mathrm{L}$ para CP con la adición de estándar interno PCB 103 (SI) en concentración $150 \mu \mathrm{g} / \mathrm{L}$, usando como solvente n-hexano. Los análisis se realizaron en un cromatógrafo de gases con detector de micro captura electrónica, 7890A, Agilent Technologies, usando las condiciones cromatográficas optimizadas para este análisis.
Para llevar a cabo la DLLME se utilizó una mezcla extractante compuesta por $20 \mathrm{~mL}$ de metanol (agente dispersante) y $1 \mathrm{~mL}$ de $\mathrm{CS}_{2}$ (agente extractante). Para los análisis se consideró un blanco y una solución acuosa que contenía CP en concentración de $250 \mu \mathrm{g} / \mathrm{L}$. Para el proceso de extracción se utilizaron tubos de vidrio con tapa, en los cuales se adicionó $5 \mathrm{~mL}$ de la solución 
de CP. Luego se adicionó $1 \mathrm{~mL}$ de la solución extractante. Luego estas mezclas se llevaron a agitación, donde se realizó la optimización del tiempo de agitación, considerando 5 tiempos entre 5 y 30 minutos (análisis en cuadriplicado). Posterior a esto las mezclas fueron centrifugadas a 2000 rpm, durante 3 minutos. La gota formada fue extraída con una pipeta Pasteur y depositada en un vial color ámbar, para luego ser llevada a sequedad con una corriente de $\mathrm{N}_{2}$ a $40{ }^{\circ} \mathrm{C}$ en baño seco. Finalmente, se realizó la reconstitución de las muestras con $0,5 \mathrm{~mL}$ de $\mathrm{n}$-hexano con adición de SI, para ser analizadas por GC- $\mu$ ECD. Los análisis se realizaron por triplicado.
Se determinó el límite de detección $\left(L_{D}\right)$ y de cuantificación $\left(L_{C}\right)$ por medio de la inyección de diez blancos. Para el $L_{D}$ se usó la fórmula $\mathrm{Y}_{\mathrm{LD}}=\mathrm{Y}_{\mathrm{B}}+3 \sigma_{\mathrm{B}}$, donde $\sigma_{\mathrm{B}}$ es la desviación estándar de las áreas obtenidas para el blanco y $\mathrm{Y}_{\mathrm{B}}$ es el promedio de área de los blancos. Para el cálculo de $\mathrm{L}_{\mathrm{C}}$ se hace el mismo procedimiento, pero se multiplica por diez la desviación estándar $\left(\mathrm{Y}_{\mathrm{LC}}=\mathrm{Y}_{\mathrm{B}}+10 \sigma_{\mathrm{B}}\right)$. Posteriormente estos valores $\left(\mathrm{Y}_{\mathrm{LD}}\right.$ y $\left.\mathrm{Y}_{\mathrm{LC}}\right)$ se interpolaron en la curva de calibración, obteniéndose la concentración correspondiente para $L_{D}$ y $L_{C}$. La precisión en este estudio fue evaluada por medio de la repetibilidad y la exactitud se evaluó por cálculos de porcentajes de recuperación del analito.

\section{RESULTADOS}

Los resultados obtenidos del análisis por GC- $\mu \mathrm{ECD}$, dieron cuenta de un tiempo de retención promedio para $\mathrm{CP}$ de 8,80 minutos, mientras que para el SI fue 9,196 minutos. Las condiciones cromatográficas utilizadas se presentan en la Tabla 1.
Tabla 1. Condiciones cromatográficas usadas para el análisis de CP por medio de GC- $\mu$ ECD

\begin{tabular}{cc}
\hline Temperatura inyección & $250{ }^{\circ} \mathrm{C}$ \\
\hline Detector & $300{ }^{\circ} \mathrm{C}$ \\
\hline Gas & $\mathrm{N}_{2}$ \\
\hline Make Up $\mathrm{N}_{2}$ & $20 \mathrm{~mL} / \mathrm{min}$ \\
\hline Flujo & $1 \mathrm{~mL} / \mathrm{min}$ \\
\hline Volumen de inyección & $10 \mu \mathrm{L}$ \\
\hline Modo de inyección & Splitless \\
\hline Columna & $\mathrm{HP}-5: 325^{\circ} \mathrm{C}:$ \\
& $30 \mathrm{~m} \times 320 \mu \mathrm{m} \times 0,25 \mu \mathrm{m}$ \\
\hline
\end{tabular}


Para el análisis se utilizó un programa de temperatura (Tabla 2) el cual considera tres rampas, desde 100 hasta $280{ }^{\circ} \mathrm{C}$, con un tiempo total de 15 minutos.

Tabla 2. Programa de temperatura usado para el análisis de CP por medio de GC- $\mu E C D$

\begin{tabular}{ccccc}
\hline Puntos & $\begin{array}{c}\text { Razón } \\
{\left[{ }^{\circ} \mathrm{C} / \mathrm{min}\right]}\end{array}$ & $\begin{array}{c}\text { Temp } \\
{\left[{ }^{\circ} \mathrm{C}\right]}\end{array}$ & $\begin{array}{c}\text { Tiempo } \\
{[\mathrm{min}]}\end{array}$ & $\begin{array}{c}\text { Tiempo } \\
\text { total } \\
{[\mathrm{min}]}\end{array}$ \\
\hline Inicial & - & 100 & 1 & 1 \\
\hline Rampa 1 & 85 & 150 & 0 & 1,5 \\
\hline Rampa 2 & 25 & 200 & 0 & 3,6 \\
\hline Rampa 3 & 7 & 280 & 0 & 15 \\
\hline
\end{tabular}

Con respecto a la curva de calibración de CP con adición de SI (Figura 2), los resultados mostraron una buena correlación en base a la linealidad de la curva $(\mathrm{Y}=0,7712$ $X-0,0792, r=0,997)$.

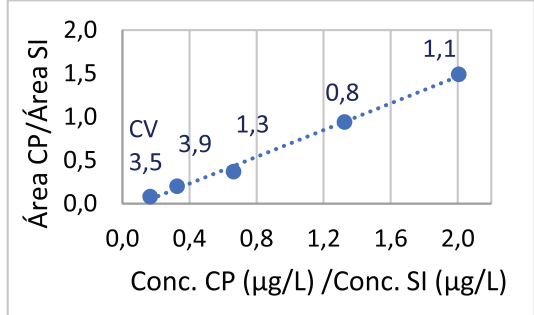

Figura 2. Curva de calibración para CP $\left(\mathrm{Y}=\right.$ Área $_{\mathrm{CP}} /$ Área $\left._{\mathrm{Sl}} ; \mathrm{X}=[]_{\mathrm{CP}} /[]_{\mathrm{SI}_{1}}\right)$
Los $L_{D}$ y $L_{C}$ obtenido fueron de 3,2 y $6,8 \mu \mathrm{g} / \mathrm{L}$, respectivamente, con una precisión expresada como la desviación estándar relativa (RSD) de $16,2 \%$.

Se analizaron los extractos de los blancos obtenidos de la DLLME, donde se determinó que no hay señales de la matriz que dificulten la cuantificación de los analitos en los tiempos de retención determinados por medio de GC- $\mu$ ECD (Figura 3).

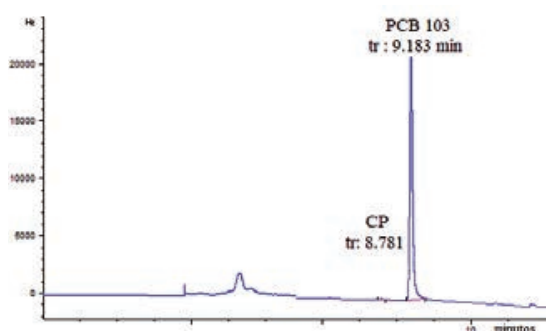

Figura 3. Cromatograma optimizado de la muestra blanco 
Los resultados de las pruebas de DLLME considerando distintos tiempos de agitación, para la extracción de CP en muestra acuosa $(250 \mu \mathrm{g} / \mathrm{L})$ se muestran en la Figura 4, donde se puede observar que el mayor porcentaje de recuperación de CP se obtiene para los tiempos de agitación 10 y 15 minutos con 108 y $88 \%$, respectivamente.

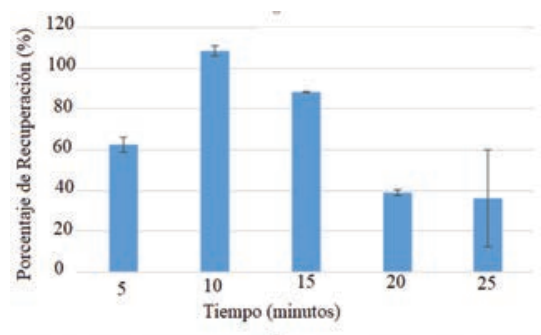

Figura 4. Relación entre porcentaje de recuperación de CP y el tiempo de agitación usada en DLLME
Los resultados dan cuenta de una precisión expresada como desviación estándar relativa menor a $6 \%$ para los tiempos menores a $20 \mathrm{mi}$ nutos. Con respecto a la obtención de la gota, los volúmenes usados permitieron obtener una gota de tamaño adecuado para su separación desde la solución de extracción y su posterior cuantificación.

\section{DISCUSIÓN}

Al comparar los $L_{D}$ y $L_{C}$ obtenidos con los reportados en literatura, se evidencia que la metodología de DLLME con GC- $\mu$ ECD tiene una alta sensibilidad, con $L_{D}$ y $L_{C}$ menores a los reportados en literatura para esta metodología de extracción (Tabla 3), esto se debe a que el detector de microcaptura electrónica posee una mayor sensibilidad, sumado a que la metodología de DLLME obtuvo porcentajes de recuperación adecuados para el análisis de este tipo de contaminante en una matriz acuosa. Al comparar los $L_{C}$ y $L_{D}$ obtenidos por DLLME y SPE del analito desde matriz acuosa, esta última metodología permite cuantificar al analito a una 
concentración dos veces menor que para DLLME. La discrepancia entre los resultados se puede deber a las diferencias en los procedimientos de las metodologías empleadas, ya que la SPE arrastra una menor cantidad de posibles interferentes, permitiendo así tener menor ruido por efecto matriz y por lo tanto discriminar a una menor concentración la señal del analito (Lazic et al., 2012; Marsin et al., 2011). La DLLME presentó una precisión expresada como de la deviación estándar relativa menor a 14,2 \%, y una exactitud expresada como la recuperación, para un tiempo de agitación de 15 minutos de $88 \%$.

Existe una preocupación por el desarrollo de nuevas metodologías de extracción, que no sólo permitan la adecuada determinación de contaminantes de interés desde matrices medioambientales, sino que además se ajuste a lo que se conoce como química analítica verde y sustentable, que implica poco impacto ambiental, bajos efectos nocivos en los operarios y asequible (Marcinkowska et al., 2019).
Tabla 3. Comparación de $L_{D} y L_{C}$ obtenido para la determinación de CP usando SPE y DLLME

\begin{tabular}{cccc}
\hline [ $\mu \mathrm{g} / \mathrm{L}]$ & $\begin{array}{c}\text { SPE } \\
\text { Lazic et al. } \\
(2012)\end{array}$ & $\begin{array}{c}\text { DLLME } \\
\text { Este, } \\
\text { trabajo }\end{array}$ & $\begin{array}{c}\text { DLLME } \\
\text { Marsin et al., } \\
(2011)\end{array}$ \\
\hline $\mathrm{L}_{\mathrm{D}}$ & 4 & 18,3 & 95 \\
$\mathrm{~L}_{\mathrm{C}}$ & 10 & 22,4 & 318 \\
\hline
\end{tabular}

La DLLME es una metodología con un bajo consumo de solventes, con pocas etapas de proceso, lo que disminuye los tiempos de análisis, a diferencia de la SPE donde se utilizan mayores volúmenes de solvente, y que considera más de una etapa, como es el acondicionamiento de las columnadas de extracción, carga de la muestra, lavado, y posterior elución del analito. Ante este escenario la metodología de DLLME optimizada en este trabajo es una alternativa que se ajusta al concepto de química analítica verde, con cifras de mérito adecuadas para el análisis de muestras medioambientales. 


\section{CONCLUSIÓN}

La metodología DLLME usando relativa menor al 14,2 \%, y un porcomo solución extractante metanol: centaje de recuperación 88 \%. De $\mathrm{CS}_{2}$ en una proporción 20:1, tiempo esta manera se puede concluir que de agitación de 15 minutos, permitió DLLME asociado a GC- $\mu$ ECD es una extraer al analito desde la matriz alternativa con cifras de mérito adeacuosa. Se determinó para la DLLME un $\mathrm{L}_{\mathrm{D}}$ y $\mathrm{L}_{\mathrm{C}}$ de 18,3 y $22,4 \mu \mathrm{g} / \mathrm{L}$, respectivamente, una precisión expresada como desviación estándar cuadas para la cuantificación de CP desde matriz acuosa, con la ventaja de ser una metodología con menor impacto ambiental que la SPE.

\section{AGRADECIMIENTOS}

Este trabajo fue financiado por Fondecyt, proyecto No. 11110223.

\section{LISTA DE REFERENCIAS}

Armenta, S., Garrigues, S., Esteve-Turrillas, F. \&, de la Guardia, M. (2019). Green extraction techniques in green analytical chemistry. TrAC Trends in Analytical Chemistry, 116, 248-253.

ATSDR. 1997. Resumen de salud pública: clorpirifos. [en línea]. <http://www.atsdr. cdc.gov/es/phs/es_phs84.pdf>. [consulta: 1 de octubre, 2021].

Environmental Protection Agency (EPA). 2016. "Revised chlorpyrifos preliminary registration review drinking Waters assessment". https://www.regulations.gov/document/EPA-HQ-OPP-2015-0653-0454 
Giesy, J., Solomon, K., Coats, J., Dixon, K., Giddings, J. \& Kenaga, E. (1999). Chlorpyrifos: Ecological Risk Assessment in North American Aquatic Environments. In: Ware G.W. (eds). Reviews of Environmental Contamination and Toxicology, vol 160. Springer, New York, NY. https://doi.org/10.1007/978-1-4612-1498-4_1

Mackay, D. (2001). Multimedia Environmental Models: The Fugacity Approach, Second Edition (2nd ed.). CRC Press. https://doi.org/10.1201/9781420032543

Marcinkowska, R., Namiesnik, J. \& Tobiszewski, M. (2019). Green and equitable analytical chemistry. Green and Sustainable Chemistry, 19, 9-23.

Márquez, S., Mosquera, R. \& Herrera, M., (2010). Estudio de la absorción y distribución del clorpirifos en plantas de pasto Kikuyo (Pennisetum clandestinum Hochst ex chiov.) cultivadas hidropónicamente. Revista Colombiana de Ciencias Pecuarias, 23(2), 158-165.

Marsin, M., Umairah, S., Akmam, M. \& Aini, W. (2011). Determination of organophosphorus pesticides by dispersive liquid-liquid microextraction coupled with gas chromatography-electron capture detection. The Malaysian Journal of Analytical Sciences, 15(2), 232-239.

Martínez, J., Pablos, M.C., Garrido, A. \& Arrebola, F.J. (2000). Pesticide trace analysis using solid-phase extraction and gas chromatography with electron-capture and tandem mass spectrometric detection in water samples. Journal of chromatography $A, 867(1), 235-245$.

Martins, M., Primel, E., Souza, S., Prestes, O., Bohrer, M. \& Zanella, R. (2012). Microextración Líquido-Líquido Dispersiva (DLLME): fundamentos y aplicaciones. Scientia Chomatographica, 4(1), 35-51.

Lambropoulou, D., Sakkas, V. \& Albanis, T. (2002). Validation of an SPME method, using PDMS, PA, PDMS-DVB, and CW-DVB SPME fiber coatings, for analysis of organophosphorus insecticides in natural waters. Analytical and bioanalytical chemistry, 374(5), 932-941.

Lazic, S., Sunjka, D., Grahovac, N., Vukovic, S. \& Jaksic, S. (2012). Determination of Chlorpyrifos in water used for agricultural production. Agriculture \& Forestry, 57(11), 17-25. 
Rezaee, M., Assadi, Y., Hosseini, M.R.M., Aghaee, E., Ahmadi, F. \& Berijani, S. (2006). Determination of organic compounds in water using dispersive liquid-liquid microextraction. Journal of Chromatography A, 1116(1), 1-9.

Rezaee, M., Yamini, Y. \& Faraji, M. (2010). Evolution of dispersive liquid-liquid microextraction method. Journal of Chromatography A, 1217(16), 2342-2357. 